\title{
Microwave based planar gas sensor using graphene and conducting polymer incorporated with double split ring resonator
}

\author{
Prakrati Azad ${ }^{1}$, M.J. Akhtar ${ }^{1,2}$ and Kamal K Kar ${ }^{1,3 *}$ \\ ${ }^{1}$ Materials Science Programme, Indian Institute of Technology Kanpur, Kanpur-208016, India \\ ${ }^{2}$ Department of Electrical Engineering, Indian Institute of Technology Kanpur, Kanpur-208016, India \\ ${ }^{3}$ Advanced Nanoengineering Materials Laboratory, Department of Mechanical Engineering, Indian \\ Institute of Technology Kanpur, Kanpur-208016, India \\ *Email: kamalkk@iitk.ac.in
}

In this work, the microwave frequency range gas sensor is proposed. The design of the sensor is small electrical resonator i.e. Double Split Ring Resonator (DSRR) on $1 \mathrm{~mm}$ thick FR4 Substrate. This small resonator is excited by transmission line i.e. high impedance line. This resonator has a resonant frequency of $13.73 \mathrm{GHz}$ and insertion loss of $8.22 \mathrm{~dB}$ when simulated on Computer Simulation Technology-Microwave Studios (CST-MWS) and this resonant frequency changes to $13.85 \mathrm{GHz}$ and insertion loss of $7.60 \mathrm{~dB}$ when fabricated in fabrication laboratory. It works on the principle of change in the resonant frequency of DSRR when gas comes in contact with gas sensing material i.e. PEDOT: PSS with graphene. The variation in the frequency (i.e. with and without gas) is caused due to change in the conductivity of the conducting polymer i.e. PEDOT: PSS and Graphene when gas is allowed in the chamber. This shift in the resonant frequency is measured by measuring S-parameter i.e. $\mathrm{S}_{21}$ (insertion loss). PEDOT: PSS is a very effective candidate for gas sensing application in microwave range because of high conductivity and change in conductivity when comes in contact with gas.

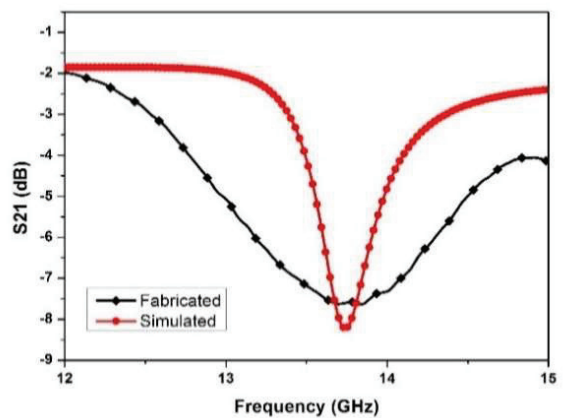

Figure 2: Insertion loss $\left(\mathrm{S}_{21}\right) \mathrm{v} / \mathrm{s}$ Frequency $(\mathrm{GHz})$ comparison between simulated and fabricated DSRR

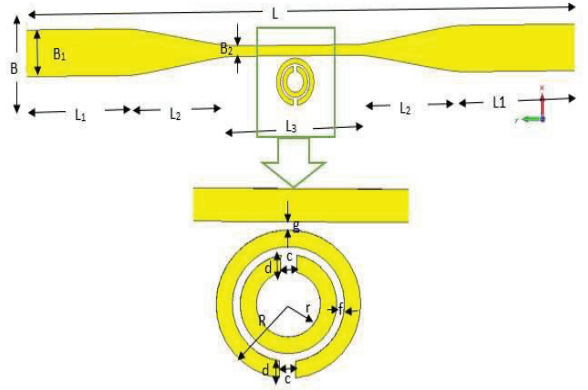

Figure 2: Electrically small resonator design

(a) High impedance transmission line i.e. $\mathrm{B}=15 \mathrm{~mm}$, $\mathrm{B}_{1}=1.78 \mathrm{~mm}, \quad \mathrm{~B}_{2}=0.4 \mathrm{~mm}, \quad \mathrm{~L}=30 \mathrm{~mm}, \mathrm{~L}_{1}=7 \mathrm{~mm}$, $\mathrm{L}_{3}=6 \mathrm{~mm}, \mathrm{~L}_{2}=5 \mathrm{~mm}$

(b) DSRR dimensions: $\mathrm{g}=\mathrm{c}=\mathrm{d}=0.2 \mathrm{~mm}, \mathrm{f}=0.1 \mathrm{~mm}$, $\mathrm{r}=0.4 \mathrm{~mm}, \mathrm{R}=0.9 \mathrm{~mm}$.

\section{References}

1. Byung-Hyun Kima, Yong-Joo Lee, Hee-Jo Lee, Yunseog Hong, and Jong- Gwan Yook, Moon Hyun Chung, Wonseok Cho, and Hyang Hee Choi, "A Gas Sensor Using Double Split-Ring Resonator Coated With Conducting Polymer at Microwave Frequencies", SENSORS, IEEE (2014), 1815.

2. S. Chopra, A. Pham, J. Gaillard, A. M. Rao, "Development of RF Carbon Nanotube Resonant Circuit Sensors for Gas Remote Sensing Applications", Microwave Symposium Digest, 2002 IEEE MTT-S International, Seattle, WA, USA, 2002, vol.2, pp. 639-642 